\title{
BELLWETHERS FOR BOUNDEDNESS OF COMPOSITION OPERATORS ON WEIGHTED BANACH SPACES OF ANALYTIC FUNCTIONS
}

\author{
PAUL S. BOURDON \\ (Received 21 June 2007; accepted 12 December 2007) \\ Communicated by J. J. Koliha
}

\begin{abstract}
Let $\mathbb{D}$ be the open unit disc, let $v: \mathbb{D} \rightarrow(0, \infty)$ be a typical weight, and let $H_{v}^{\infty}$ be the corresponding weighted Banach space consisting of analytic functions $f$ on $\mathbb{D}$ such that $\|f\|_{v}:=\sup _{z \in \mathbb{D}} v(z)|f(z)|<$ $\infty$. We call $H_{v}^{\infty}$ a typical-growth space. For $\varphi$ a holomorphic self-map of $\mathbb{D}$, let $C_{\varphi}$ denote the composition operator induced by $\varphi$. We say that $C_{\varphi}$ is a bellwether for boundedness of composition operators on typical-growth spaces if for each typical weight $v, C_{\varphi}$ acts boundedly on $H_{v}^{\infty}$ only if all composition operators act boundedly on $H_{v}^{\infty}$. We show that a sufficient condition for $C_{\varphi}$ to be a bellwether for boundedness is that $\varphi$ have an angular derivative of modulus less than 1 at a point on $\partial \mathbb{D}$. We raise the question of whether this angular-derivative condition is also necessary for $C_{\varphi}$ to be a bellwether for boundedness.
\end{abstract}

2000 Mathematics subject classification: primary 47B33; secondary 46E15.

Keywords and phrases: composition operator, weighted Bergman space of infinite order, boundedness, angular derivative, Lusky condition.

\section{Introduction}

Let $H(\mathbb{D})$ denote the collection of holomorphic functions on the open unit disc $\mathbb{D}$, let $\varphi$ denote an element of $H(\mathbb{D})$ such that $\varphi(\mathbb{D}) \subseteq \mathbb{D}$, and let $C_{\varphi}$ be the composition operator induced by $\varphi$, so that whenever $f$ is a function defined on $\mathbb{D}, C_{\varphi} f$ is the function defined on $\mathbb{D}$ by $\left(C_{\varphi} f\right)(z)=f(\varphi(z))$. A typical weight on the open unit disc $\mathbb{D}$ is a continuous strictly-positive function $v$ on $\mathbb{D}$ that is radial, is nonincreasing with respect to $|z|$, and satisfies $\lim _{|z| \rightarrow 1^{-}} v(z)=0$. The associated typical-growth space $H_{v}^{\infty}$ is defined by

$$
H_{v}^{\infty}=\left\{f \in H(\mathbb{D}):\|f\|_{v}:=\sup _{z \in \mathbb{D}} v(z)|f(z)|<\infty\right\} .
$$

(C) 2009 Australian Mathematical Society 1446-7887/2009 \$16.00 
The norm $\|\cdot\|_{v}$ gives $H_{v}^{\infty}$ a Banach-space structure. Note that convergence in $H_{v}^{\infty}$ implies uniform convergence on compact subsets of $\mathbb{D}$. Applying the closed-graph theorem, one sees that $C_{\varphi}$ is bounded on $H_{v}^{\infty}$ if and only if it maps $H_{v}^{\infty}$ into itself.

As a example, consider

$$
v_{\mathrm{e}}(z)=\exp (-1 /(1-|z|)), \quad \psi(z)=(1+z) / 2 \quad \text { and } \quad \varphi(z)=1 /(2-z) .
$$

Note that $f(z)=\exp (1 /(1-z)) \in H_{v_{\mathrm{e}}}^{\infty}$ but $f(\psi(z))=\exp (2 /(1-z)) \notin H_{v_{\mathrm{e}}}^{\infty}$, so that $C_{\psi}$ is not bounded on $H_{v_{\mathrm{e}}}^{\infty}$. On the other hand, for any $f \in H_{v_{\mathrm{e}}}^{\infty}$ and $z \in \mathbb{D}$,

$$
\begin{aligned}
v_{\mathrm{e}}(z)|f(\varphi(z))| & =\frac{v_{\mathrm{e}}(z)}{v_{\mathrm{e}}(\varphi(z))} v_{\mathrm{e}}(\varphi(z))|f(\varphi(z))| \\
& \leq \exp \left(\frac{1}{1-|1 /(2-z)|}-\frac{1}{1-|z|}\right)\|f\|_{v_{\mathrm{e}}} \\
& \leq \exp \left(\frac{1}{1-1 /(2-|z|)}-\frac{1}{1-|z|}\right)\|f\|_{v_{\mathrm{e}}} \\
& =\exp (1)\|f\|_{v_{\mathrm{e}}}
\end{aligned}
$$

and thus $C_{\varphi}$ is bounded on $H_{v_{\mathrm{e}}}^{\infty}$.

In [4], Bonet et al. provide characterizations of boundedness for composition operators on $H_{v}^{\infty}$, some of which do not require that the weight $v$ be typical. When $v$ is typical, they show that if $C_{\varphi}$ is bounded on $H_{v}^{\infty}$ for some disc automorphism $\varphi$ such that $\varphi(0) \neq 0$ then all composition operators are bounded on $H_{v}^{\infty}$ [4, proof of Theorem 2.3]. Thus automorphisms that don't fix the origin induce composition operators that are bellwethers for boundedness.

DEFINITION. We say $C_{\varphi}$ is a bellwether for boundedness of composition operators on typical-growth spaces if for each typical weight $v$, the boundedness of $C_{\varphi}$ on $H_{v}^{\infty}$ ensures the boundedness of all composition operators on $H_{v}^{\infty}$.

Every disc automorphism not fixing the origin has angular derivatives of modulus less than 1. In Section 3 of this paper, we show that whenever $\varphi$ has an angular derivative of modulus less than $1, C_{\varphi}$ is a bellwether for boundedness of composition operators on typical-growth spaces. On the other hand, if all angular derivatives of $\varphi$ exceed 1 , then it is easy to see that $C_{\varphi}$ is not a bellwether for boundedness (see Corollary 3). Finally, we note that $\varphi$ having an angular derivative of 1 at a point is not sufficient to ensure that $C_{\varphi}$ is a boundedness bellwether: consider the example above where $\varphi(z)=1 /(2-z)$. The mapping $\varphi$ has angular derivative 1 at 1 , and $C_{\varphi}$ is bounded on $H_{v_{\mathrm{e}}}^{\infty}$. However, not all composition operators are bounded on $H_{v_{\mathrm{e}}}^{\infty}$; in particular, $C_{\psi}$, with $\psi(z)=(1+z) / 2$, is not. The preceding results raise the following question.

Question. If $C_{\varphi}$ is a bellwether for boundedness of composition operators on typical-growth spaces, must $\varphi$ have angular derivative less than 1 at some point of $\partial \mathbb{D}$ ? 


\section{Preliminaries}

2.1. Automorphisms of $\mathbb{D}$ As a corollary to the Schwarz lemma, if $\alpha \in H(\mathbb{D})$ is an automorphism of $\mathbb{D}$ then there must be a $p \in \mathbb{D}$ and a unimodular constant $\zeta$ such that $\alpha(z)=\zeta \alpha_{p}(z)$, where $\alpha_{p}$ is given by

$$
\alpha_{p}(z)=\frac{z-p}{1-\bar{p} z} .
$$

Observe that the inverse of $\alpha_{p}$ is $\alpha_{-p}$. A little algebra yields the following standard and useful identity:

$$
1-\left|\alpha_{p}(z)\right|^{2}=\frac{\left(1-|z|^{2}\right)\left(1-|p|^{2}\right)}{|1-\bar{p} z|^{2}} .
$$

LEMMA 1. Suppose that $\alpha$ is a holomorphic automorphism of $\mathbb{D}$. Then for $0 \leq r<1$,

$$
\max _{|z|=r}|\alpha(z)|=\frac{|\alpha(0)|+r}{1+|\alpha(0)| r} .
$$

PROOF. There is a unimodular constant $\zeta$ such that $\alpha(z)=\zeta(z-p) /(1-\bar{p} z)$. Note that $|\alpha(0)|=|p|$, and that the lemma clearly holds when $\alpha(0)=0$. Assume that $\alpha(0) \neq 0$, and apply (1), to obtain

$$
1-|\alpha(z)|^{2}=\frac{\left(1-|z|^{2}\right)\left(1-|p|^{2}\right)}{|1-\bar{p} z|^{2}} .
$$

The preceding quantity clearly achieves its minimum value on $\{z:|z|=r\}$ when $z=-r p /|p|$, and the lemma follows.

2.2. Angular derivatives Recall that every bounded function in $H(\mathbb{D})$ has nontangential limits at every point of a subset of $\partial \mathbb{D}$ having full Lebesgue measure. When $f \in H(\mathbb{D})$ has a nontangential limit at $\zeta$, we denote the value of the limit by $f(\zeta)$. The holomorphic self-map $\varphi$ of $\mathbb{D}$ has angular derivative at $\zeta \in \partial D$ provided that there is a unimodular constant $\eta$ for which

$$
\angle \lim _{z \rightarrow \zeta} \frac{\varphi(z)-\eta}{z-\zeta}
$$

exists as a complex number, where $\angle \lim _{z \rightarrow \zeta}$ denotes the nontangential limit. The limit (2) is written $\varphi^{\prime}(\zeta)$, and is called the angular derivative of $\varphi$ at $\zeta$. The following classical result provides some alternate ways to view angular derivatives.

Julia-CARATHÉOdory Theorem. Suppose that $\varphi$ is a holomorphic self-map of $\mathbb{D}$ and $\zeta \in \partial \mathbb{D}$. The following are equivalent:

(a) there exists a point $\eta$ in $\partial \mathbb{D}$ such that

$$
\angle \lim _{z \rightarrow \zeta} \frac{\varphi(z)-\eta}{z-\zeta}
$$

is finite; 
(b) both $\varphi$ and $\varphi^{\prime}$ have finite nontangential limits at $\zeta$ and $\varphi(\zeta)=\eta$ has modulus 1 ;

(c)

$$
\liminf _{z \rightarrow \zeta} \frac{1-|\varphi(z)|}{1-|z|}=\delta<\infty .
$$

Moreover, when the conditions above hold, $\delta>0$; the limit $\varphi^{\prime}(\zeta)$ in $(a)$ is also equal to $\angle \lim _{z \rightarrow \zeta} \varphi^{\prime}(z)$, and their common value is $\eta \bar{\zeta} \delta$ (thus the limit infimum of $(c)$ is equal to $\left.\left|\varphi^{\prime}(\zeta)\right|\right)$; finally, $\angle \lim _{z \rightarrow \zeta}(1-|\varphi(z)|) /(1-|z|)=\left|\varphi^{\prime}(\zeta)\right|$.

Note that if, for example, there were some sequence $\left(z_{n}\right)$ in $\mathbb{D}$ with $\left|z_{n}\right|$ approaching 1 for which $\left|\varphi\left(z_{n}\right)\right| \geq\left|z_{n}\right|$ for all $n$, then at any limit point $\zeta$ of $\left(z_{n}\right)$, the function $\varphi$ would have an angular derivative, and $\left|\varphi^{\prime}(\zeta)\right| \leq 1$. Hence we have the following easy corollary of the Julia-Carathéodory theorem.

COROLlary 2. Suppose that $\varphi$ has no angular derivatives having modulus $\leq 1$. Then there is a positive number $r<1$ such that $|\varphi(z)|<|z|$ whenever $r<|z|<1$.

Also useful to us will be the Julia-Carathéodory inequality: suppose that $\lim \inf _{z \rightarrow \zeta}(1-|\varphi(z)|) /(1-|z|)=\delta<\infty$, and $\eta$ is the nontangential limit of $\varphi$ at $\zeta$; then for all $z \in \mathbb{D}$,

$$
\frac{|\eta-\varphi(z)|^{2}}{1-|\varphi(z)|^{2}} \leq \delta \frac{|\zeta-z|^{2}}{1-|z|^{2}} .
$$

For discussions of the Julia-Carathéodory theorem and inequality, as well as their proofs, see, for example, [9, Ch. 4] or [6, Section 2.3].

2.3. Denjoy-Wolff point For each positive integer $n$, let $\varphi^{[n]}$ denote the $n$th iterate of $\varphi$, so that, for example, $\varphi^{[2]}=\varphi \circ \varphi$. The Denjoy-Wolff theorem describes the behaviour of iterate sequences for self-maps $\varphi$ of $\mathbb{D}$. Recall that a disc automorphism is called elliptic if it fixes a point in $\mathbb{D}$.

Denjoy-WolfF TheOREM. If $\varphi$ is an automorphism of $\mathbb{D}$ that is not elliptic, then there is a point $\omega$ in the closure of $\mathbb{D}$ such that

$$
\omega=\lim _{n \rightarrow \infty} \varphi^{[n]}(z)
$$

for each $z \in \mathbb{D}$.

The point $\omega$, called the Denjoy-Wolff point of $\varphi$, is also characterized as follows: if $|\omega|<1$, then $\varphi(\omega)=w$ and $\left|\varphi^{\prime}(\omega)\right|<1$; if $\omega \in \partial \mathbb{D}$, then $\varphi(\omega)=\omega$ and $0<\varphi^{\prime}(\omega) \leq 1$.

2.4. Boundedness of composition operators on $\boldsymbol{H}_{\boldsymbol{v}}^{\infty}$ We continue to assume that $v$ is a typical weight: that is, a continuous, positive, radial function that is nonincreasing with respect to $|z|$ and satisfies $\lim _{|z| \rightarrow 1^{-}} v(z)=0$. Let $\tilde{v}$ be the weight associated with $v$ defined on $\mathbb{D}$ by

$$
\tilde{v}(z)=\left(\sup \left\{|f(z)|: f \in H_{v}^{\infty},\|f\|_{v} \leq 1\right\}\right)^{-1},
$$


so that $\tilde{v}(z)=1 /\left\|\delta_{z}\right\|$ where $\delta_{z}: H_{v}^{\infty} \rightarrow \mathbb{C}$ is the linear functional of evaluation at $z \in \mathbb{D}$. It is not difficult to show that if $v$ is typical then so is $\tilde{v}$ [4, Proposition 1.1], and that $H_{v}^{\infty}=H_{\tilde{v}}^{\infty}$ with $\|\cdot\|_{v}=\|\cdot\|_{\tilde{v}}$ [2, p. 144]. The associated weight has the desirable property that for each $z \in \mathbb{D}$ there is a function $f_{z}$ in the unit ball of $H_{v}^{\infty}$ such that $\left|f_{z}(z)\right|=1 / \tilde{v}(z)$.

An easy sufficient condition for boundedness of the composition operator $C_{\varphi}$ on $H_{v}^{\infty}$ is that

$$
\sup _{z \in \mathbb{D}} \frac{v(z)}{v(\varphi(z))}<\infty .
$$

In fact, $\left\|C_{\varphi} f\right\|_{v} \leq \sup _{z \in \mathbb{D}} v(z) / v(\varphi(z))\|f\|_{v}$. Because $v$ is nonincreasing with respect to $|z|$, the preceding observation, together with the Schwarz lemma, shows that $C_{\varphi}$ is bounded on $H_{v}^{\infty}$, with $\left\|C_{\varphi}\right\|=1$ whenever $\varphi(0)=0$. Similarly, $C_{\varphi}$ is bounded if $\varphi$ has no angular derivatives of modulus less than or equal to 1: apply Corollary 2 to see that $v(z) / v(\varphi(z))$ is less than 1 on an annulus of the form $\{z: r<|z|<1\}$; because $v$ is continuous and positive and $\varphi(\{z:|z| \leq r\})$ is bounded away from $\partial \mathbb{D}$, the finiteness of (4) follows. Reference [4, Theorem 2.4] shows that the preceding two situations are the only ones in which $C_{\varphi}$ is bounded for all typical weights. Our interest is in the following simple corollary.

Corollary 3. Suppose that all angular derivatives of $\varphi$ exceed 1 . Then $C_{\varphi}$ is not a bellwether for boundedness of composition operators on typical-growth spaces.

PROOF. If all angular derivatives of $\varphi$ exceed 1 , then in particular $C_{\varphi}$ is bounded on $H_{v_{\mathrm{e}}}^{\infty}$ for the typical weight $v_{\mathrm{e}}(z)=\exp (-1 /(1-|z|))$. However, as we have seen, not all composition operators are bounded on $H_{v_{\mathrm{e}}}^{\infty}$.

The simple sufficient condition (4) becomes necessary when $v$ is replaced by its associated weight. Indeed, [4, Proposition 2.1] provides the following characterization of boundedness: $C_{\varphi}$ is bounded on $H_{v}^{\infty}$ if and only if

$$
\sup _{z \in \mathbb{D}} \frac{\tilde{v}(z)}{\tilde{v}(\varphi(z))}<\infty .
$$

This holds even when $v$ is not typical. We remark that information about norms and essential norms of composition operators on $H_{v}^{\infty}$ may be found in $[3,5,8]$, while some spectral information may be found in, for example, [1].

\section{Results}

We continue to assume that $v$ is a typical weight. Reference [4, Theorem 2.3] asserts that the following are equivalent:

(i) all composition operators $C_{\varphi}: H_{v}^{\infty} \rightarrow H_{v}^{\infty}$ are bounded;

(ii) all composition operators $C_{\varphi}: H_{v}^{0} \rightarrow H_{v}^{0}$ are bounded; 
(iii)

$$
\inf _{n \in \mathbb{N}} \frac{\tilde{v}\left(1-2^{-n-1}\right)}{\tilde{v}\left(1-2^{-n}\right)}>0 .
$$

Here $\mathbb{N}$ is the set of positive integers, and $H_{v}^{0}$ is the subspace of $H_{v}^{\infty}$ defined by $H_{v}^{0}=\left\{f \in H(\mathbb{D}): \lim _{|z| \rightarrow 1^{-}} v(z)|f(z)|=0\right\}$. We remark that the condition (5) was used by Lusky [7] in his study of the case where $H_{v}^{0}$ is isomorphic to $c_{0}$. The proof in [4] of the equivalence of (i) and (iii) is based on the observation that if $C_{\alpha_{p}}$ is bounded for every $p \in \mathbb{D}$ then all composition operators on $H_{v}^{\infty}$ are bounded. The point is that if $\varphi$ is an arbitrary self-map of $\mathbb{D}$ and $p=\varphi(0)$ then $\psi:=\alpha_{p} \circ \varphi$ induces a bounded composition operator on $H_{v}^{\infty}$ because $\psi(0)=0$. If $C_{\alpha_{-p}}$ is also bounded, then so is $C_{\varphi}=C_{\psi} C_{\alpha_{-p}}$. The proof of [4, Theorem 2.3] shows that the boundedness of $C_{\alpha}$ for a single automorphism $\alpha$ (not fixing the origin) is sufficient for (5) to hold, and that if (5) holds then all automorphisms induce bounded composition operators. We expand upon ideas in the proof of [4, Theorem 2.3] to obtain the following theorem, whose proof provides a direct argument that the boundedness of a single automorphic composition operator $C_{\alpha}$, with $\alpha(0) \neq 0$, implies the boundedness of every automorphic composition operator.

For $0<a<1$, let

$$
\psi_{a}(z)=a z+1-a,
$$

so that $\psi_{a}$ is a self-map of $\mathbb{D}$ and $\psi_{a}^{[n]}(0)=1-a^{n}$ for each $n \in \mathbb{N}$.

THEOREM 4. Let $v$ be a typical weight. The following are equivalent.

(i) $\quad C_{\varphi}: H_{v}^{\infty} \rightarrow H_{v}^{\infty}$ is bounded for every analytic self-map $\varphi$ of $\mathbb{D}$;

(ii) $C_{\psi_{a}}: H_{v}^{\infty} \rightarrow H_{v}^{\infty}$ is bounded for every $a \in(0,1)$;

(iii) $\inf _{n \in \mathbb{N}}\left(\tilde{v}\left(1-a^{n+1}\right) / \tilde{v}\left(1-a^{n}\right)\right)>0$ for every $a \in(0,1)$;

(iv) $\inf _{n \in \mathbb{N}}\left(\tilde{v}\left(1-a^{n+1}\right) / \tilde{v}\left(1-a^{n}\right)\right)>0$ for some $a \in(0,1)$;

(v) $\inf _{t \in(0,1]}(\tilde{v}(1-a t) / \tilde{v}(1-t))>0$ for some $a \in(0,1)$;

(vi) $C_{\alpha}: H_{v}^{\infty} \rightarrow H_{v}^{\infty}$ is bounded for some automorphism $\alpha$ of $\mathbb{D}$ with $\alpha(0) \neq 0$;

(vii) $C_{\alpha}: H_{v}^{\infty} \rightarrow H_{v}^{\infty}$ is bounded for every automorphism $\alpha$ of $\mathbb{D}$.

Proof. That (i) implies (ii) is trivial. Suppose that (ii) holds and $a \in(0,1)$. Then by [4, Proposition 2.1], there is a positive constant $M$ such that

$$
\frac{\tilde{v}(z)}{\tilde{v}\left(\psi_{a}(z)\right)}<M \quad \forall z \in \mathbb{D} .
$$

Letting $z=\psi_{a}^{[n]}(0)$ in the preceding inequality, where $n \in \mathbb{N}$ is arbitrary, and rearranging,

$$
\frac{\tilde{v}\left(1-a^{n+1}\right)}{\tilde{v}\left(1-a^{n}\right)}>\frac{1}{M} .
$$

Because $a \in(0,1)$ and $n \in \mathbb{N}$ are arbitrary, we deduce (iii). 
That (iii) implies (iv) is trivial. Now suppose that (iv) holds, and that the infimum is $\beta>0$. Because $\tilde{v}$ is nonincreasing with respect to $|z|$, if $a \leq t \leq 1$ then

$$
\gamma:=\frac{\tilde{v}\left(1-a^{2}\right)}{\tilde{v}(0)} \leq \frac{\tilde{v}(1-a t)}{\tilde{v}(1-t)} .
$$

This $\gamma$ is a positive constant, since $\tilde{v}$ is a positive function on $\mathbb{D}$. Now let $k$ denote a positive integer, and assume that $a^{k+1} \leq t<a^{k}$. Then

$$
\frac{\tilde{v}(1-a t)}{\tilde{v}(1-t)} \geq \frac{\tilde{v}\left(1-a^{k+2}\right)}{\tilde{v}\left(1-a^{k}\right)}=\frac{\tilde{v}\left(1-a^{k+2}\right)}{\tilde{v}\left(1-a^{k+1}\right)} \frac{\tilde{v}\left(1-a^{k+1}\right)}{\tilde{v}\left(1-a^{k}\right)} \geq \beta^{2} .
$$

We see that for any $t \in(0,1]$,

$$
\frac{\tilde{v}(1-a t)}{\tilde{v}(1-t)} \geq \min \left\{\gamma, \beta^{2}\right\}
$$

so that (v) holds.

Suppose (v) holds, with the infimum being $\lambda>0$. Let $p=(1-a) /(1+a)$, so that $p$ is positive and $(1-p) /(1+p)=a$. We show that the automorphism $\alpha_{p}(z)=$ $(z-p) /(1-p z)$ induces a bounded composition operator. Note that $\alpha_{p}(0) \neq 0$ because $p \neq 0$. Using Lemma 1 and the fact that $\tilde{v}$ is nonincreasing and radial, we find that for $z \in \mathbb{D}$,

$$
\begin{aligned}
\frac{\tilde{v}(z)}{\tilde{v}\left(\alpha_{p}(z)\right)} & \leq \frac{\tilde{v}(|z|)}{\tilde{v}((p+|z|) /(1+p|z|))} \\
& =\frac{\tilde{v}(1-(1-|z|))}{\tilde{v}(1-(1-(p+|z|) /(1+p|z|)))} \\
& =\frac{\tilde{v}(1-(1-|z|))}{\tilde{v}(1-(1-p)(1-|z|) /(1+p|z|))} \\
& \leq \frac{\tilde{v}(1-(1-|z|))}{\tilde{v}(1-((1-p) /(1+p))(1-|z|))} \quad(\tilde{v} \text { is nonincreasing }) \\
& =\frac{\tilde{v}(1-(1-|z|))}{\tilde{v}(1-a(1-|z|))} \\
& \leq 1 / \lambda .
\end{aligned}
$$

It follows that $C_{\alpha_{p}}$ is bounded [4, Proposition 2.1], and we see that (v) implies (vi).

Suppose that (vi) holds: $C_{\alpha}: H_{v}^{\infty} \rightarrow H_{v}^{\infty}$ is bounded for $\alpha=\zeta \alpha_{p}$ where $|\zeta|=1$ and $p \in \mathbb{D} \backslash\{0\}$. Because $C_{\alpha}$ is bounded,

$$
\infty>\sup _{z \in \mathbb{D}} \tilde{v}(z) / \tilde{v}(\alpha(z))=\sup _{z \in \mathbb{D}} \tilde{v}(z) / \tilde{v}\left(\alpha_{p}(z)\right),
$$

where the final equality holds because $\tilde{v}$ is radial. Thus $C_{\alpha_{p}}$ is also bounded. Note that $\alpha_{p}$ has Denjoy-Wolff point $-p /|p| \in \partial \mathbb{D}$; hence $\left|\alpha_{p}^{[n]}(0)\right| \rightarrow 1$ as $n \rightarrow \infty$. Let 
$\tau(z)=\xi(z-q) /(1-\bar{q} z)$ be an arbitrary disc automorphism. Choose the positive integer $n$ so that $\left|\alpha_{p}^{[n]}(0)\right|>|q|$, and set $s=\left|\alpha_{p}^{[n]}(0)\right|$. We know that $C_{\alpha_{p}^{[n]}}$ is bounded. Thus there is a constant $C$ such that for every $z \in \mathbb{D}$,

$$
\frac{\tilde{v}(z)}{\tilde{v}\left(\alpha_{p}^{[n]}(z)\right)} \leq C
$$

Let $z \in \mathbb{D}$ be arbitrary, let $r=|z|$, and choose $z_{0}$ with $\left|z_{0}\right|=r$ so that $\left|\alpha_{p}^{[n]}\left(z_{0}\right)\right|=$ $(s+r) /(1+s r)$ (Lemma 1). Because $x \mapsto(x+r) /(1+x r)$ is increasing for $-1 / r<x<\infty,|q|<s$, and $\tilde{v}$ is nonincreasing,

$$
\frac{\tilde{v}(z)}{\tilde{v}(\tau(z))} \leq \frac{\tilde{v}(r)}{\tilde{v}((|q|+r) /(1+|q| r))} \leq \frac{\tilde{v}(r)}{\tilde{v}((s+r) /(1+s r))}=\frac{\tilde{v}\left(z_{0}\right)}{\tilde{v}\left(\alpha^{[n]}\left(z_{0}\right)\right)} \leq C,
$$

and it follows that $C_{\tau}$ is bounded. Thus, (vi) implies (vii). We have already indicated why (vii) implies (i); see the comments following equation (5) above. Hence the proof is complete.

Note that the proof of the preceding theorem shows that for each $a \in(0,1)$ the composition operator $C_{\psi_{a}}$ induced by $\psi_{a}(z)=a z+1-a$ is a bellwether for boundedness: if $C_{\psi_{a}}$ is bounded on the typical-growth space $H_{v}^{\infty}$, then

$$
\inf _{n \in \mathbb{N}} \tilde{v}\left(1-a^{n+1}\right) / \tilde{v}\left(1-a^{n}\right)>0
$$

so that $C_{\varphi}$ is bounded on $H_{v}^{\infty}$ for all $\varphi$. That $C_{\psi_{a}}$ is a bellwether for boundedness also follows from the next result because the angular derivative of $\psi_{a}$ at 1 is $a<1$.

Theorem 5. Let $v$ be typical weight. Suppose that $C_{\varphi}: H_{v}^{\infty} \rightarrow H_{v}^{\infty}$ is bounded and that $\varphi$ has angular derivative less than 1 at some point $\zeta \in \partial \mathbb{D}$. Then every composition operator on $H_{v}^{\infty}$ is bounded.

Proof. By the Julia-Carathéodory theorem, there is an $\eta \in \partial \mathbb{D}$ such that $\varphi$ has nontangential limit $\eta$ at $\zeta$. Because the composition operators $C_{\bar{\eta} z}$ and $C_{\zeta z}$ are bounded, the composition operator with symbol $\psi(z)=\bar{\eta} \varphi(\zeta z)$ is also bounded. Moreover, $\psi(1)=1$ and $\psi^{\prime}(1)=\bar{\eta} \varphi^{\prime}(\zeta) \zeta=\left|\varphi^{\prime}(\zeta)\right|<1$. Thus we see that $C_{\psi}$ is bounded and that $\psi$ has Denjoy-Wolff point 1 with $a:=\psi^{\prime}(1)<1$.

Applying the Julia-Carathéodory inequality (3) inductively, we see that for every $n \in \mathbb{N}$,

$$
\frac{\left|1-\psi^{[n]}(z)\right|^{2}}{1-\left|\psi^{[n]}(z)\right|^{2}} \leq a^{n} \frac{|1-z|^{2}}{1-|z|^{2}}
$$

Thus, for each $n \in \mathbb{N}, 1-\left|\psi^{[n]}(0)\right| \leq 2 a^{n}$. Hence, for $n \in \mathbb{N}$,

$$
1-\left|\psi^{[n]}(0)\right|=g(n) a^{n},
$$


where $g$ is a positive bounded function on $\mathbb{N}$. Because $\left(\psi^{[n]}(0)\right)$ converges nontangentially to 1 (see, for example, [6, Lemma 2.66, p. 82]), we may apply the Julia-Carathéodory theorem to conclude that

$$
\lim _{n \rightarrow \infty} \frac{1-\left|\psi^{[n+1]}(0)\right|}{1-\left|\psi^{[n]}(0)\right|}=a .
$$

Equivalently,

$$
\lim _{n \rightarrow \infty} \frac{g(n+1)}{g(n)}=1
$$

For each $n \in \mathbb{N}$, set $e_{n}=n+\log _{a}(g(n))$. Then

$$
e_{n+1}-e_{n}=1+\log _{a}\left(\frac{g(n+1)}{g(n)}\right) \text {. }
$$

Since $\log _{a}(g(n+1) / g(n))$ approaches 0 as $n \rightarrow \infty$, there is a natural number $K$ such that whenever $n \geq K$, the gap between $e_{n+1}$ and $e_{n}$ exceeds $1 / 2$. Because $C_{\psi}$ is bounded, $\left(C_{\psi}\right)^{3}=C_{\psi[3]}$ is also bounded, and there is a constant $M$ such that

$$
\frac{\tilde{v}(|z|)}{\tilde{v}\left(\left|\psi^{[3]}(z)\right|\right)} \leq M \quad \forall z \in \mathbb{D} .
$$

In particular, for every $n \in \mathbb{N}$,

$$
M \geq \frac{\tilde{v}\left(1-\left(1-\left|\psi^{[n]}(0)\right|\right)\right)}{\tilde{v}\left(1-\left(1-\left|\psi^{[n+3]}(0)\right|\right)\right)}=\frac{\tilde{v}\left(1-a^{e_{n}}\right)}{\tilde{v}\left(1-a^{e_{n+3}}\right)} .
$$

Let $j \in \mathbb{N}$ exceed $K+\log _{a}(g(K))=e_{K}$. Let $n_{0} \geq K$ be the greatest positive integer such that $e_{n_{0}} \leq j$. Note that $e_{n_{0}+1}>j$. Because the gap between $e_{n+1}$ and $e_{n}$ exceeds $1 / 2$ for every $n \geq K$, we have $e_{n_{0}+3}>e_{n_{0}+1}+1 \geq j+1$. Because $\tilde{v}$ is nonincreasing,

$$
\frac{\tilde{v}\left(1-a^{j}\right)}{\tilde{v}\left(1-a^{j+1}\right)} \leq \frac{\tilde{v}\left(1-a^{e_{n_{0}}}\right)}{\tilde{v}\left(1-a^{e_{n_{0}+3}}\right)} \leq M,
$$

and it follows, since $j \geq e_{K}$ is arbitrary, that

$$
\inf _{n \in \mathbb{N}} \frac{\tilde{v}\left(1-a^{n+1}\right)}{\tilde{v}\left(1-a^{n}\right)}>0 .
$$

Thus (iv) of Theorem 4 holds, and all composition operators on $H_{v}^{\infty}$ are bounded.

Using the Julia-Carathéodory theorem, we can summarize our results (Theorem 5 and Corollary 3) on bellwethers for boundedness as follows. Let

$$
\liminf _{|z| \rightarrow 1^{-}} \frac{1-|\varphi(z)|}{1-|z|}=\delta
$$

(where we allow $\delta=\infty$ ). If $\delta<1$, then $C_{\varphi}$ is a bellwether for boundedness of composition operators on typical-growth spaces; if $\delta>1$, then $C_{\varphi}$ is not a boundedness bellwether.

The obvious question is whether $\delta<1$ is necessary for $C_{\varphi}$ to be a bellwether for boundedness. 


\section{Acknowledgement}

The author wishes to thank Joel Shapiro for helpful discussions concerning the material presented in this paper.

\section{References}

[1] R. Aron and M. Lindström, 'Spectra of weighted composition operators on weighted Banach spaces of analytic functions', Israel J. Math. 141 (2004), 263-276.

[2] K. D. Bierstedt, J. Bonet and J. Taskinen, 'Associated weights and spaces of holomorphic functions', Studia Math. 127 (1998), 137-168.

[3] J. Bonet, P. Domański and M. Lindström, 'Essential norm and weak compactness of composition operators on weighted Banach spaces of analytic functions', Canad. Math. Bull. 42 (1999), 139-148.

[4] J. Bonet, P. Domański, M. Lindström and J. Taskinen, 'Composition operators between weighted Banach spaces of analytic functions', J. Austral. Math. Soc. (Series A) 64 (1998), 101-118.

[5] M. D. Contreras and A. G. Hernandez-Diaz, 'Weighted composition operators in weighted Banach spaces of analytic functions', J. Austral. Math. Soc. (Series A) 69 (2000), 41-60.

[6] C. Cowen and B. MacCluer, Composition Operators on Spaces of Analytic Functions (CRC Press, Boca Raton, FL, 1995).

[7] W. Lusky, 'On weighted spaces of harmonic and holomorphic functions', J. London Math. Soc. 51 (1995), 309-320.

[8] A. Montes-Rodríguez, 'Weighted composition operators on weighted Banach spaces of analytic functions', J. London Math. Soc. 61 (2000), 872-884.

[9] J. H. Shapiro, Composition Operators and Classical Function Theory (Springer-Verlag, Berlin, 1993).

PAUL S. BOURDON, Department of Mathematics, Washington and Lee University, Lexington, VA 24450, USA

e-mail: pbourdon@wlu.edu 